Original article

\title{
Caloric restriction alleviates alpha-synuclein toxicity in aged yeast cells by controlling the opposite roles of Tor1 and Sir2 on autophagy
}

\author{
Ana Guedes $^{\mathrm{a}, \mathrm{b}}$, Paula Ludovico ${ }^{\mathrm{a}, \mathrm{b}, *}$, Belém Sampaio-Marques ${ }^{\mathrm{a}, \mathrm{b}}$ \\ a Life and Health Sciences Research Institute (ICVS), School of Health Sciences, University of Minho, Braga, Portugal \\ b ICVS/3B's - PT Government Associate Laboratory, Braga, Guimarães, Portugal
}

\section{A R T I C L E I N F O}

\section{Article history:}

Received 14 March 2016

Received in revised form 11 April 2016

Accepted 18 April 2016

Available online 21 April 2016

\section{Keywords:}

Caloric restriction

Autophagy

Sirtuins

Alpha-synuclein

Synucleinopathies

\begin{abstract}
A B S T R A C T
Alpha-synuclein (syn) is the main component of proteinaceous inclusions known as Lewy bodies (LBs), which are implicated in the pathogenesis of the neurodegenerative diseases known as synucleinopathies, like Parkinson's disease (PD). Aging is a major risk factor for PD and thus, interventions that delay aging will have promising effects in PD and other synucleinopathies. Caloric restriction (CR) is the only nongenetic intervention shown to promote lifespan extension in several model organisms. CR has been shown to alleviate syn toxicity and herein we confirmed the same effect on the yeast model for synucleinopathies during chronological lifespan. The data gathered showed that TOR1 deletion also results in similar longevity extension and abrogation of syn toxicity. Intriguingly, these interventions were associated with decreased autophagy, which was maintained at homeostatic levels. Autophagy maintenance at homeostatic levels promoted by CR or TOR1 abrogation in syn-expressing cells was achieved by decreasing Sir2 levels and activity. Furthermore, the opposite function of Tor1 and Sir2 in autophagy is probably associated with the maintenance of autophagy activity at homeostatic levels, a central event linked to abrogation of syn toxicity promoted by $C R$.
\end{abstract}

(c) 2016 Elsevier Ireland Ltd. All rights reserved.

\section{Introduction}

$\alpha$-Synuclein (syn) is a major component of Lewy bodies (LB), the hallmark protein inclusions made up of insoluble and fibrillar forms of syn (Spillantini et al., 1998), implicated in the pathology of several neurodegenerative diseases known as synucleinopathies, such as Parkinson disease (PD). Since the discovery of the involvement of syn in PD, a whole range of model systems have been developed to study syn toxicity. One of the most powerful and versatile cellular models to explore the molecular properties and the cellular dysfunction associated with synucleinopathies is the bakers' yeast Saccharomyces cerevisiae. Although S. cerevisiae lacks an orthologue of syn, heterologous expression of syn results in toxicity and cell death (Outeiro and Lindquist, 2003). Moreover, several of the pathways that mediate syn toxicity in yeast cells were found conserved

\footnotetext{
Abbreviation: CLS, chronological lifespan; CR, caloric restriction; CFUs, counting colony-forming units; NCR, non caloric restriction; syn, $\alpha$-synuclein.

* Corresponding author at: Life and Health Sciences Research Institute (ICVS), School of Health Sciences, University of Minho, Campus de Gualtar, 4710-057 Braga, Portugal.

E-mail address: pludovico@ecsaude.uminho.pt (P. Ludovico).
}

in other eukaryotic models for synucleinopathies (Franssens et al., 2010; Tenreiro et al., 2013).

Caloric restriction (CR), without malnutrition, is the most effective and reproducible physiological intervention promoting longevity from yeast to mammals [reviewed in (Fontana and Partridge, 2015)]. It was already demonstrated that CR could alleviate syn toxicity in different PD models, such as Caenorhabditis elegans, mice and primates (Cohen et al., 2004; Duan and Mattson, 1999; Jadiya et al., 2011; Maswood et al., 2004; Mladenovic et al., 2007; Vartiainen et al., 2006), however, the molecular mechanisms involved are still not well understood. It is well established that $\mathrm{CR}$ intervention modulates the nutrient/metabolic-sensing pathways, including sirtuins, the target of rapamycin (TOR), and AMP-activated protein kinase (AMPK), to promote longevity (Jadiya et al., 2011; Maswood et al., 2004; Zhang et al., 2011). Furthermore, mounting evidence suggests that autophagy is a downstream mechanism required for the longevity promoted by $\mathrm{CR}$, as already demonstrated in several models, like yeast, $C$. elegans and human cell lines (Alvers et al., 2009; Jia and Levine, 2007; Morselli et al., 2010). Autophagy has a recognized central role in cellular homeostasis maintenance, since physiological levels of autophagy act as a cytoprotective process during various stress conditions, and in contrast excessive or uncontrolled levels of autophagy are able to induce autophagy-dependent cell death (Sampaio-Marques et al., 
Table 1

Strains and plasmids employed in this study.

\begin{tabular}{|c|c|c|}
\hline Yeast Strain & Genotype & Source \\
\hline BY4741 & $\begin{array}{l}\text { MATa his } 3 \Delta 1 \\
\text { leu } 2 \Delta 0 \text { met } 15 \Delta 0 \\
\text { ura } 3 \Delta 0\end{array}$ & Euroscarf \\
\hline BY4741 pho8 $\Delta$ & $\begin{array}{l}\text { MAT a his3D1 } \\
\text { leu2D0 met15D0 } \\
\text { ura3D0 pho8:HPH }\end{array}$ & $\begin{array}{l}\text { (Sampaio-Marques } \\
\text { et al., 2012) }\end{array}$ \\
\hline $\operatorname{sir} 2 \Delta$ & $\begin{array}{l}\text { MATa his3D1 } \\
\text { leu2D0 met15D0 } \\
\text { ura3D0 } \\
\text { sir2:kanMX4 }\end{array}$ & Euroscarf \\
\hline $\operatorname{tor} 1 \Delta$ & $\begin{array}{l}\text { MATa his } 3 \Delta 1 \\
\text { leu } 2 \Delta 0 \text { met } 15 \Delta 0 \\
\text { ura } 3 \Delta 0 \\
\text { tor1:kanMX4 }\end{array}$ & Euroscarf \\
\hline Plasmids & Type of Plasmid & Source \\
\hline pYX222 & $2 \mu$ & $\begin{array}{l}\text { (Sampaio-Marques } \\
\text { et al., 2012) }\end{array}$ \\
\hline pYX222- syn & $2 \mu$ & $\begin{array}{l}\text { (Sampaio-Marques } \\
\text { et al., 2012) }\end{array}$ \\
\hline pYX242-cytPho8 & $2 \mu$ & (Mendl et al., 2011) \\
\hline pRS416-GFPAtg8 & $2 \mu$ & (Mari et al., 2010) \\
\hline
\end{tabular}

2011). Furthermore, sirtuins and the TOR pathway form a complex contrasting network, in which autophagy is one of the downstream effectors, suggesting that modulation of sirtuins and TOR may, at least partly, be associated with the autophagy-dependent lifespan extension promoted by CR [reviewed in (Blagosklonny, 2010)]. Interestingly, these players that are implicated in the CR-mediated longevity have also been assigned as modulators of syn toxicity (Crews et al., 2010; Decressac et al., 2013; Donmez et al., 2012; Sampaio-Marques et al., 2012; van Ham et al., 2008; Yeger-Lotem et al., 2009), but the precise contribution of each, as well as the crosstalk between them, in the context of synucleinopathies, is still elusive. Herein, the effects of CR on syn-mediated toxicity were explored in yeast cells during chronological lifespan (CLS). Our data showed that CR increased the CLS of cells expressing syn. Furthermore, data also suggested that $\mathrm{CR}$ effects are associated with decreased Tor 1 and Sir 2 activities. The crosstalk between Tor 1 and Sir2 in the context of synucleinopathies relies in the maintenance of autophagy at homeostatic levels, which appears to be a central phenomenon linked to abrogation of syn toxicity promoted by CR.

\section{Materials and methods}

\subsection{Strains and media}

The yeast strains and plasmids used in this study are listed in Table 1. Cells stocks were maintained in YEPD agar medium containing $0.5 \%$ yeast extract, $1 \%$ peptone, $2 \%$ agar, and $2 \%$ glucose. All experiments were performed in synthetic complete (SC) medium containing glucose as a carbon source and $0.67 \%$ yeast nitrogen base without amino acids (Difco Laboratories) supplemented with excess amino acids and bases for which the strains were auxotrophic $(50 \mu \mathrm{g} / \mathrm{mL}$ histidine, $100 \mathrm{mg} / \mathrm{L}$ methionine, $300 \mu \mathrm{g} / \mathrm{mL}$ leucine and $100 \mu \mathrm{g} / \mathrm{mL}$ uracil).

\subsection{Chronological lifespan}

Caloric restriction (CR) was accomplished by reducing the glucose concentration from 2 to $0.5 \%$ or $0.05 \%$ in the initial culture medium. Overnight cultures were grown in SC medium containing the two different concentrations of glucose and then inoculated into flasks containing medium with the same concentration of glucose at a volume ratio of $1: 3$. These cultures were then incubated at $26^{\circ} \mathrm{C}$ with shaking at $150 \mathrm{rpm}$. Cultures reached stationary phase 2-3 days later and this was considered day 0 of CLS. Survival was assessed by counting colony-forming units (CFUs) after 2 days of incubation of culture aliquots at $26^{\circ} \mathrm{C}$ on YEPD agar plates beginning at day 0 of CLS (when viability was considered to be $100 \%$ ) and then again every $2-3$ days until less than $0.01 \%$ of the cells in the culture were viable.

\subsection{Autophagy activity}

The monitoring of autophagy was performed according to the protocol described by Noda and Klionsky (Noda and Klionsky, 2008). Briefly, strains deleted for $\mathrm{PHO} 8$ gene were co-transformed with cytPho8 plasmid. At specific time points, $5 \times 10^{8}$ cells were collected, harvested, washed in $2 \mathrm{~mL}$ of ice-cold water containing $0.85 \% \mathrm{NaCl}, 1 \mathrm{mM}$ PMSF, and resuspended in $300 \mu \mathrm{L}$ lysis buffer ( $20 \mathrm{mM}$ PIPES, $0.5 \%$ Triton $\mathrm{X}-100,50 \mathrm{mM} \mathrm{KCl}, 100 \mathrm{mM}$ potassium

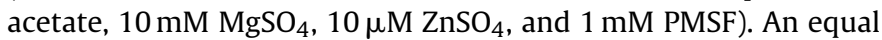
volume of acid-washed glass beads was added and the cells were lysed by vortexing for $7 \mathrm{~min}$. To start the assay, $100 \mu \mathrm{L}$ of extract was added to $400 \mu \mathrm{L}$ reaction buffer [ $250 \mathrm{mM}$ Tris- $\mathrm{HCl}$, $\mathrm{pH} 8.5,0.4 \%$ Triton X-100, $10 \mathrm{mM}$ MgSO4, 4 mM nitrophenyl phosphate (Sigma, N9389)], and samples were incubated $15 \mathrm{~min}$ at $37^{\circ} \mathrm{C}$ before terminating the reaction by adding $500 \mu \mathrm{L}$ of stop buffer (2 M glycine, $\mathrm{pH}$ 11). Evolution of nitrophenol was monitored by measuring absorbance at $405 \mathrm{~nm}$ using a microplate reader Model680 (BioRad), and each sample was corrected with the time 0 blank. Protein concentration in the extracts was measured with the Bradford (BioRad) according to the manufacturer's instructions.

\subsection{Preparation of protein extracts and western blot analysis}

For detection of protein levels by western-blot, the total cellular extracts were collected at specific time points and extracted as previously described (Zhang et al., 2011). Briefly, cells were pretreated with $2 \mathrm{M}$ lithium acetate for $5 \mathrm{~min}$ at room temperature. After lithium acetate removal, $0.4 \mathrm{M} \mathrm{NaOH}$ were added for $5 \mathrm{~min}$ on ice. Next, the cell were resuspended in SDS-PAGE sample buffer and boiled for $5 \mathrm{~min}$. Of total protein, $20 \mu \mathrm{g}$ were resolved on a $12 \%$ SDS gel and transferred to a nitrocellulose membrane during $7 \mathrm{~min}$ at $25 \mathrm{~V}$ in trans-Blot Turbo ${ }^{\circledR}$ transfer system. Membranes were blocked with tris buffered saline (TBS) with $0.1 \%$ Tween 20 (TBST) containing 5\% skim milk, followed by incubation with primary antibodies against: anti- $\alpha$-syn (1:1000, Sigma); anti-GFP (1:5000; Abcam); anti-acetyl-Histone H3 (Lys9) (1:5000, Millipore); antiHistone H3 (1:5000, abcam); anti-Sir2 (1:5000); anti-actin (1:5000, kindly provide by Dr. Gourlay, C) and anti-PGK (1:5000; Invitrogen) in TBST containing $1 \%$ skim milk and primary antibody. After washing with TBS, the membranes were incubated with the respective secondary antibody, HRP-conjugated anti-rabbit IgG, anti-mouse IgG or anti-goat IgG at a dilution of 1:5000 in 1\% skim milk. Protein levels were detected after incubation with SuperSignal West Femto Maximum Sensitivity Substrate (Thermo Scientific) or Clarity Western ECL Substrate (Bio-Rad). Digital images of the western blotting were obtained in a ChemiDoc XRS System (Bio-Rad) with Quantity One (Bio-Rad) software.

\subsection{Statistical analysis}

The results shown are mean values and standard error of the mean of at least three independent biological replicates. Statistical analyses were determined using two-way ANOVA. A $p$-value of less than 0.05 was considered as a significant difference. 

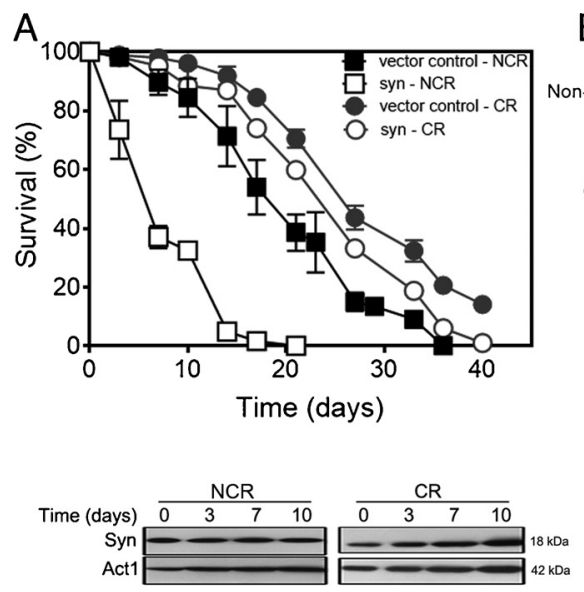
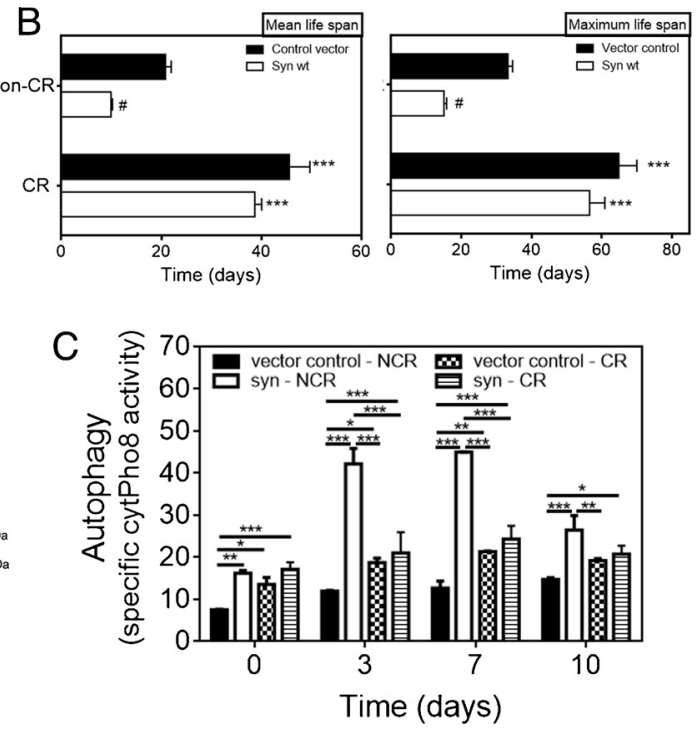

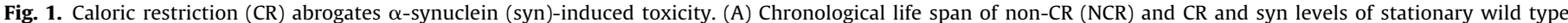

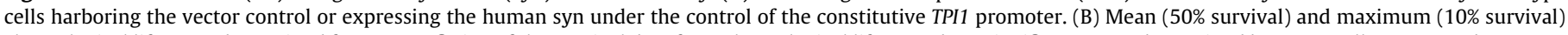

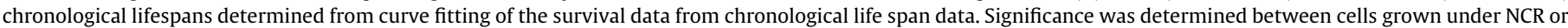

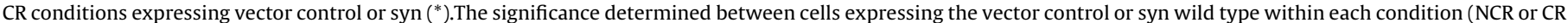

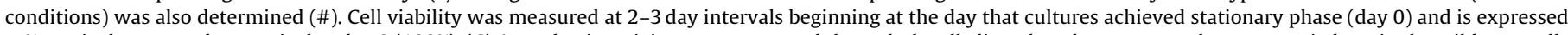

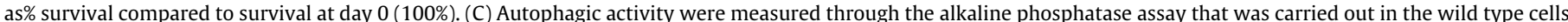

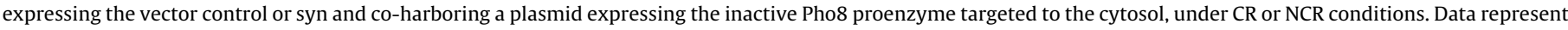

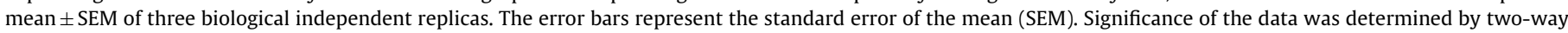
ANOVA $\left({ }^{* * *} \mathrm{p}<0.001 ;{ }^{*} \mathrm{p}<0.001\right)$.

\section{Results}

\subsection{Caloric restriction abrogates $\alpha$-synuclein-mediated toxicity through autophagy modulation}

Caloric restriction (CR), or reduction of calorie intake without malnutrition, is one of the most robust interventions associated with aging delay, in diverse species from yeast to mammals (Fontana et al., 2010). This evidence led to the suggestion that $\mathrm{CR}$ might also protect against the development of age-related diseases, including neurodegenerative disease, such as Parkinson's disease (PD). Consistently, CR has been shown to increase lifespan of different PD models (Duan and Mattson, 1999; Maswood et al., 2004; Vartiainen et al., 2006). To the best of our knowledge, CR effects on yeast model for synucleinopathies [yeast cells expressing $\alpha$-synuclein (syn)] were never addressed. Therefore, we decided to explore, the CR contribution for modulation of syn toxicity in yeast cells during chronological lifespan (CLS). To evaluate CR effects on syn toxicity, a yeast model constitutively expressing syn under the control of an endogenous triose phosphate isomerase (TPI) promoter from a single integrated copy was used and CLS was monitored under non-CR (NCR) (medium with $2 \%$ of glucose) or CR (medium with $0.5 \%$ of glucose) conditions (Mesquita et al., 2010; Sampaio-Marques et al., 2012). The results showed that CR intervention was able to promote an exacerbated CLS extension of syn-expressing cells similar to CLS displayed by vector controlwild type cells (Fig. 1A). The determination of the mean $(50 \%$ survival) and maximum ( $10 \%$ survival) CLS confirmed that CR intervention resulted in an increased mean and maximum lifespan of syn-expressing cells (Fig. 1B). Notably, CR more than doubles the mean and maximum CLS of cells expressing syn (Fig. 1B). Altogether, this data suggests that CR abrogates syn toxicity observed during CLS (Fig. 1A, B).

The pro-longevity mechanisms associated with CR have not been definitively determined. Mounting evidence suggests that induction of autophagy, a physiological process of lysosomal/vacuolar-dependent recycling, is one of the CR-longevity mediators (Aris et al., 2013; Morselli et al., 2010). Given that our previous work demonstrated that, in aged yeast cells expressing syn, increased autophagy is associated with syn toxicity and consequently with premature aging (Sampaio-Marques et al., 2012), the relationship between $\mathrm{CR}$, syn-induced toxicity and autophagy was investigated. For such purpose, $\mathrm{PHO} 8$ mutant cells expressing syn or harboring the vector control were co-transformed with a plasmid expressing an inactive Pho8 proenzyme targeted to the cytosol, to assess autophagy (Noda and Klionsky, 2008). Under CR intervention, syn-expressing cells presented significantly lower autophagy activity than that displayed by same cells under NCR conditions (Fig. 1C). Nevertheless, CR intervention promotes itself a significant increase of autophagic activity when vector control-cells in NCR and CR conditions are compared (Fig. 1C), suggesting that CR is able to modulate autophagy to homeostatic levels. Although CR promotes an abrogation of syn toxicity associated with modulation of autophagy, this effect is not associated with alteration of the syn levels, since they are similar and independent of CR intervention (Fig. 1A). We have previously shown that abrogation of mitophagy results in decreased syn toxicity while the syn levels and the number of syn foci were maintained unaltered (SampaioMarques et al., 2012). Taken together, this data suggests that $C R$ maintains autophagy at physiological levels leading to the abrogation of syn-toxicity and consequent CLS extension.

\subsection{Abrogation of TOR1 mimics the caloric restriction effects on $\alpha$-synuclein toxicity through autophagy modulation}

The reduction of the activity of TOR/Sch9 and Ras/PKA nutrientsensing pathways is a central determinant for life-extending effects of CR (Dilova et al., 2007; Fontana et al., 2010; Kaeberlein et al., 2005). Thus, we wondered whether TOR1 deletion is sufficient to abrogate syn toxicity independently of other putative CR beneficial effects. The results obtained showed that in NCR conditions, deletion of TOR 1 increased the CLS of cells expressing syn to values 
A
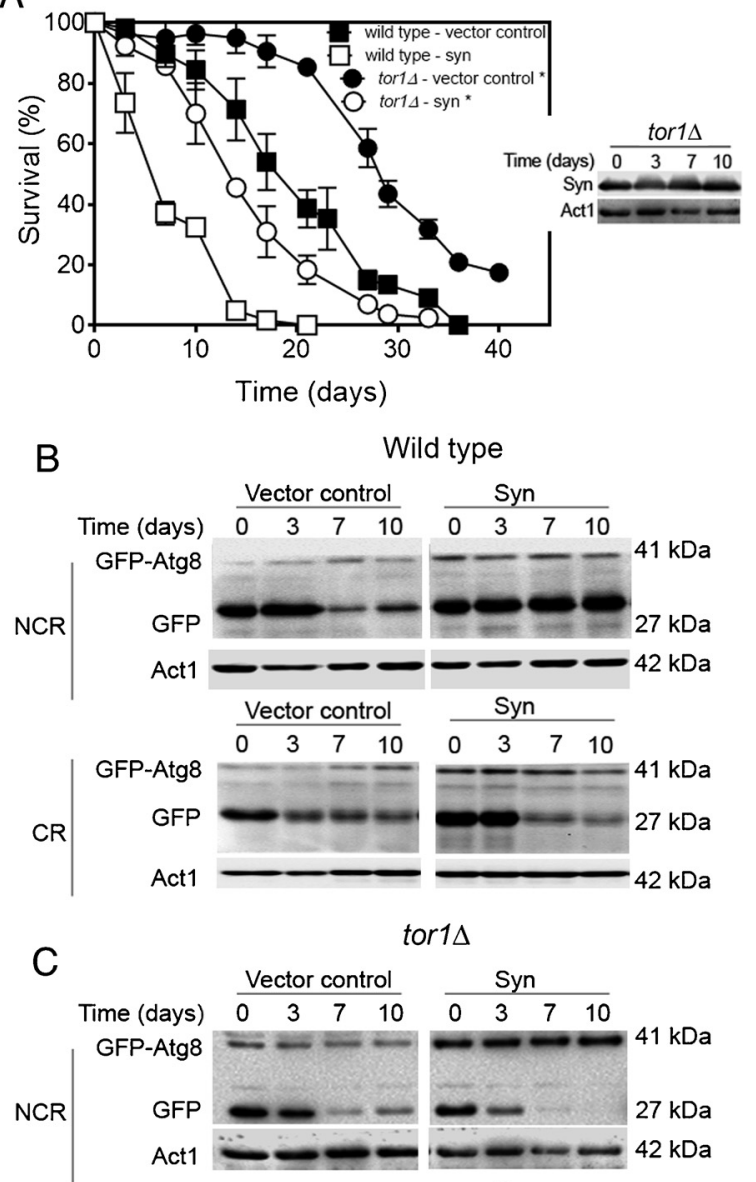

Fig. 2. TOR1 abrogation mimics caloric restriction (CR) effects on $\alpha$-synuclein (syn)expressing cells through autophagy regulation. (A) Chronological life span and syn levels of wild type and tor1 $\Delta$ cells harboring the vector control or expressing the human syn under the control of the constitutive TPI1 promoter, under non-CR (NCR). Significance was determined between wild type and tor $1 \Delta$ cells expressing vector control or syn $\left({ }^{*}\right)$.Cell viability was measured at 2-3 day intervals beginning at the day that cultures achieved stationary phase (day 0 ) and is expressed as\% survival compared to survival at day $0(100 \%)$. Autophagy activity (B) of wild type cells submitted to non-CR (NCR) or CR conditions and (C) tor1 $\Delta$ cells under NCR conditions. Autophagy activity was evaluated by the GFP-Atg8 processing assay, immunoblotting analysis with antibodies against GFP, in BY4742 and tor1 $\Delta$ cells. GFP-Atg8 and GFP bands are labeled. Data represent mean \pm SEM of three biological independent replicas. The error bars represent the standard error of the mean (SEM). Significance of the data was determined by two-way ANOVA ( $\mathrm{p} p<0.001)$.

similar to those obtained for vector control wild type cells (Fig. 2A). This data suggests that TOR1 deletion abrogates the syn toxicity as observed by CR.

Given that exacerbated autophagy mediates syn-induced toxicity and that TOR pathway is a central regulator of autophagy, we decided to evaluate autophagy activity in TOR1 deleted expressing syn, by the GFP-Atg8 processing assay. This assay is based on the vacuolar proteolysis of GFP-Atg8 that results in the release of intact GFP, resistant to degradation in yeast, and is directly correlated with the autophagic flux (Cheong and Klionsky, 2008). Surprisingly, autophagy, was found to be decreased in TOR1 deleted cells expressing syn, under NCR conditions, when compared to wild type cells expressing syn (Fig. 2C). Furthermore, TOR1 deleted cells expressing syn displayed an autophagy activity pattern similar to that obtained for wild type cells expressing syn, under CR conditions (Fig. 2B, C). Altogether the results indicated that modulation of autophagy to homeostatic levels promoted by CR in cells expressing syn is dependent on CR inhibitory effects on TOR pathway. These results are quite puzzling because an inhibition of TOR pathway will natural result in the increase of autophagy and not a decrease, as observed in syn-expressing cells. Therefore, we decided to evaluate other regulators of autophagy such as sirtuin 2 (Sir2) and its relation with TOR pathway.

\subsection{Tor1 inhibits Sir2 activity, the main regulator of autophagy in syn-expressing cells}

Although the nutrient sensing players have a central role in the CR-mediating CLS extension, the metabolic sensor Sir2 was also linked with the longevity effects promoted by CR (Lin et al., 2000; Wood et al., 2004). Nevertheless, the relationship between CR and Sir2 during CLS remains controversial. We have previously shown that deletion of SIR2 does not have a major impact on CLS but it abrogates syn toxicity through modulation of autophagy (SampaioMarques et al., 2014, 2012). Furthermore, some evidence supports that sirtuins and TOR can be involved in the same longevity pathway (Medvedik et al., 2007). Nevertheless, both have opposing effects on autophagy. Active Tor1 inhibits autophagy, while active Sir2 stimulates autophagy (Madeo et al., 2009; Morselli et al., 2010).

Keeping in mind the role of CR, Sir2 and Tor1 on the modulation of autophagy, we decided to investigate the relationship between them during CLS in the yeast model for synucleinopathies. For such purpose, the Sir2 protein levels were evaluated by immunoblot analysis in wild type cells, during CLS under CR conditions. Data revealed that under CR conditions Sir2 levels are decreased (Fig. 3A, B), from day 0 until day 10 of CLS, suggesting that CR regulates Sir2 levels during CLS. Nevertheless, under CR conditions, synexpressing cells still display increased Sir2 levels, at days 3 and 7 of CLS, in comparison with the vector control cells (Fig. 3A, B), compatible with the known role of Sir2 in syn toxicity (Buttner et al., 2010; Sampaio-Marques et al., 2012). To complement the above results, Sir2 activity was also determined in the same conditions. Sir2 promotes histone deacetylation, including the histone 3 at lysine 9 (H3K9) (Vaquero, 2009), which is an indirect measurement of Sir2 activity. We therefore analyzed the effects promoted by $\mathrm{CR}$ on the level of histone 3 acetylation by using antibodies that specifically detect acetylated H3K9. Increased H3K9 acetylated levels inversely correlated with Sir2 deacetylase activity. Consistently with the determined Sir2 levels, CR decreases Sir2 activity as reflected by the higher levels of histone H3K9 acetylation (Fig. 3C, D). Once again, it is observed that syn-expressing cells presented increased Sir2 activity levels in comparison with the vector control cells (Fig. 3C, D).

Next, the levels and activity of Sir2 were also assessed in tor $1 \Delta$ cells expressing syn, during CLS under NCR conditions. Data showed that TOR1 abrogation leads to a decrease in the Sir2 levels when compared with wild type cells submitted to the same conditions (Fig. 3A, B). Moreover, it is revealed that the Sir2 levels displayed by TOR1 deleted cells are similar to that showed by the wild type cells submitted to CR conditions (Fig. 3A, B). Consistently, tor $1 \Delta$ cells expressing syn presented decreased Sir2 activity in comparison with wild type cells under NCR conditions and similar activity in comparison with the cells submitted to CR (Fig. 3C, D).

Together, data suggests that in wild type cells expressing syn, CR leads to the down regulation of Tor 1 and Sir2 activities. The data obtained in tor $1 \Delta$ cells suggests that Tor1 is a major regulator of Sir2 activity. The hierarchic relationship between Tor 1 and Sir2 and the known opposite roles of these two players on autophagy apparently keeps autophagy at homeostatic levels in syn-expressing cells. This mechanism is most probably underlying the benefic effects of $\mathrm{CR}$, dependent on autophagy, on syn toxicity. 

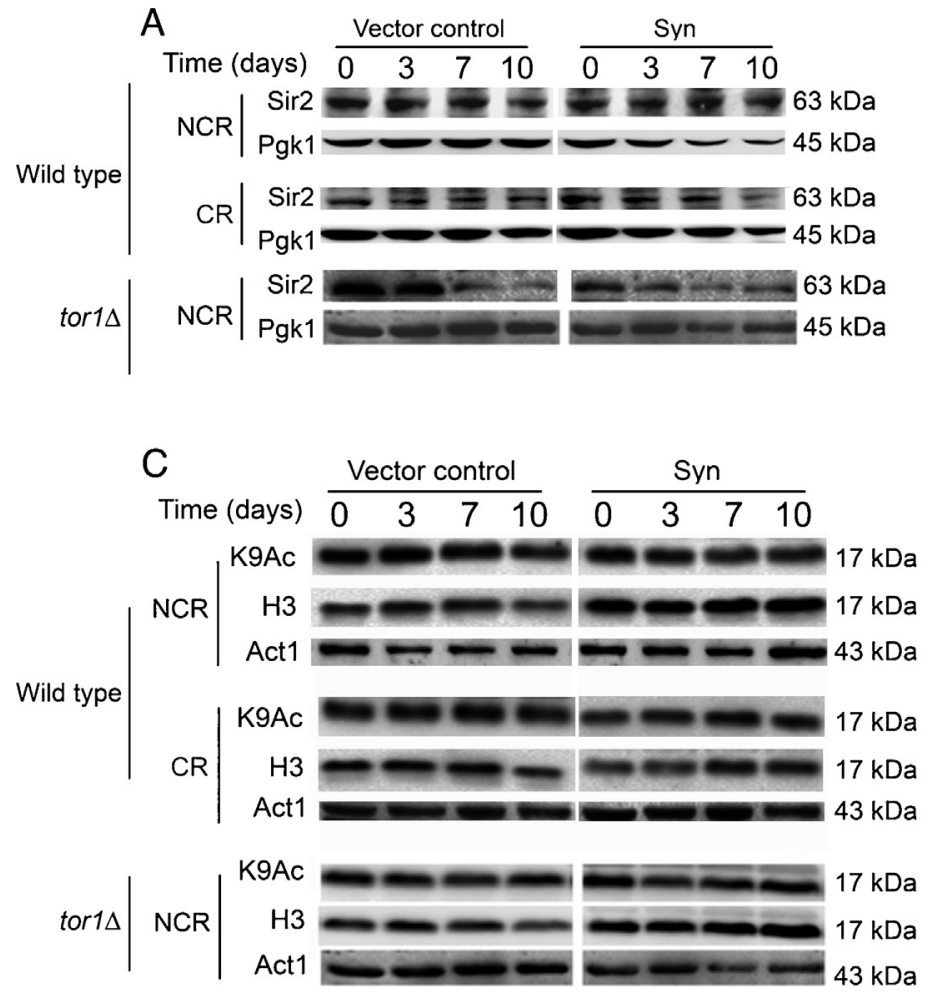
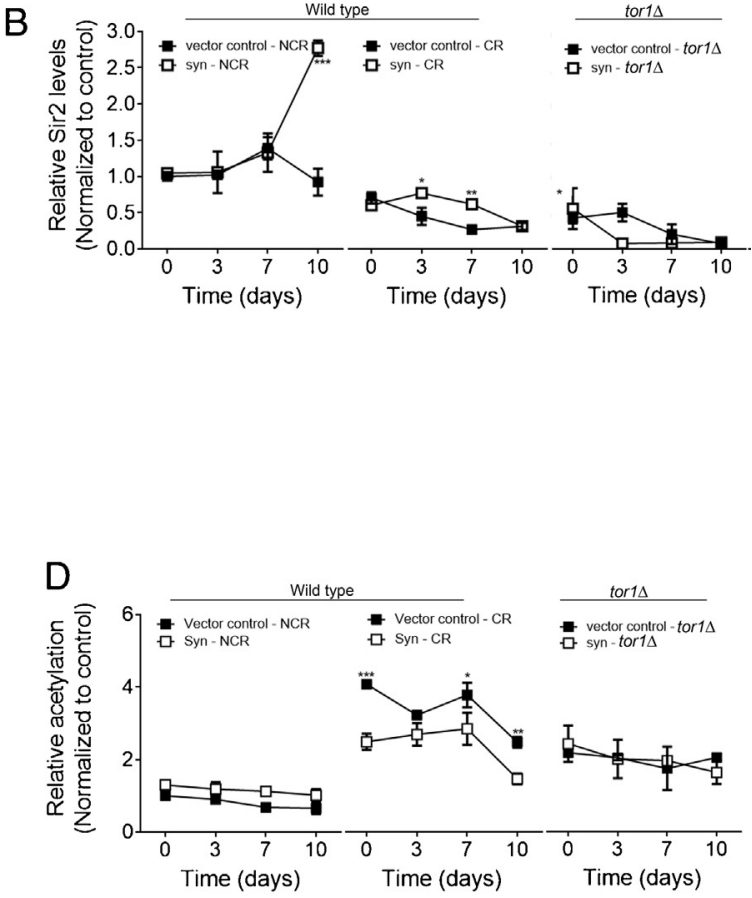

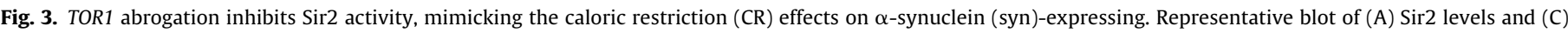

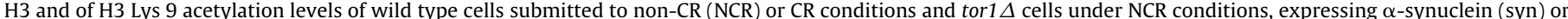

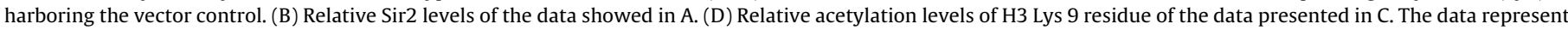

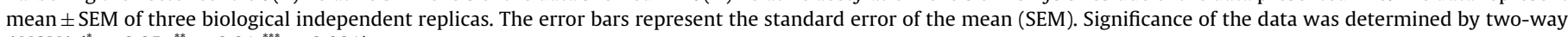
ANOVA $\left({ }^{*} \mathrm{p}<0.05 ;{ }^{* *} \mathrm{p}<0.01 ;{ }^{* * *} \mathrm{p}<0.001\right)$.

\section{Discussion}

Synucleinopathies, as Parkinson's disease (PD), are currently incurable disorders that affect a growing number of individuals. Therefore, the identification of new strategies for therapeutic intervention are of utmost importance. Aging is one of the main risk factors for the development of these disorders. Thus, it is expectable that interventions underlying aging delay might be an effective strategy. The only non-genetic intervention able to delay aging is caloric restriction (CR) (Speakman and Mitchell, 2011). Here, using a yeast model for synucleinopathies, we found that CR promotes chronological lifespan (CLS) extension of aged yeast cells heterologous expressing the human $\alpha$-synuclein (syn). This is in accordance with previous studies using different models for PD, such as C. elegans, mice or primates, evidencing that $\mathrm{CR}$ intervention provide protection and extends lifespan (Cohen et al., 2004; Duan and Mattson, 1999; Jadiya et al., 2011; Maswood et al., 2004; Mladenovic et al., 2007; Vartiainen et al., 2006).

Aging is commonly associated with decreased autophagy flux that is apparently counterbalanced by CR intervention (Cuervo, 2008). Data herein present showed that autophagy induction and flux, assessed by GFP-Atg8 processing assay, is different in vector control cells under NCR and CR conditions. It is clear that independently of autophagy induction levels, $C R$ is able to decrease the autophagy flux to homeostatic levels (Fig. 2B). This seems more evident in vector control cells where CR is able to sustain a constant autophagy flux during CLS in contrast to NCR conditions, in agreement with the protective role of autophagy under CR conditions. Moreover, syn expression promoted an induction of autophagy, as shown by GFP-Atg8 levels, regulated by the endogenous ATG8 promoter, independently of NCR or CR condition. The increased
CLS promoted by CR was found to be associated with the maintenance of autophagy at homeostatic levels, which corresponds to a decrease of autophagy in syn-expressing cells when submitted to $\mathrm{CR}$, in comparison with same cells under NCR. We, and others, have demonstrated that syn-induced toxicity is associated with exacerbated autophagy (Choubey et al., 2011; Sampaio-Marques et al., 2012; Stefanis et al., 2001; Xilouri et al., 2009). Therefore, the data herein presented indicates that $\mathrm{CR}$ is not only able to control the exacerbated autophagic levels displayed by syn-expressing cells, but is also capable to maintain the autophagy at homeostatic levels, essential for cellular homeostasis (Liu and Levine, 2015). The elucidation of the molecular mechanisms underlying CR control of autophagy at homeostatic levels in a syn scenario is therefore of utmost importance.

One of the mechanisms elicited by CR relies is the modulation of the nutrient/metabolic-sensing pathways, including TOR and sirtuins (Dilova et al., 2007). In this study, we showed that in yeast cells expressing syn, TOR1 abrogation promotes an extension of CLS similarly to that observed in wild type cells under CR intervention, suggesting that $\mathrm{CR}$ mediated inhibition of TOR is sufficient to rescue cells from syn toxicity. Our observations are in agreement with those described in other studies demonstrating that TOR inhibition is correlated with decreased syn toxicity and neurodegeneration (Crews et al., 2010; Decressac et al., 2013; Yeger-Lotem et al., 2009). Surprisingly, TOR1 abrogation resulted in the same autophagic phenotype as observed with CR intervention and thus the role of Sir2 was also explored.

There are many intriguing links among sirtuins, TOR, CR and longevity. CR slows aging in several models, at least in part, by down regulating the activity of TOR. Furthermore, it has been proposed, as an evolutionarily conserved mechanism, that CR leads to the activation of sirtuins (Boily et al., 2008; Cohen et al., 2004; Lin et al., 


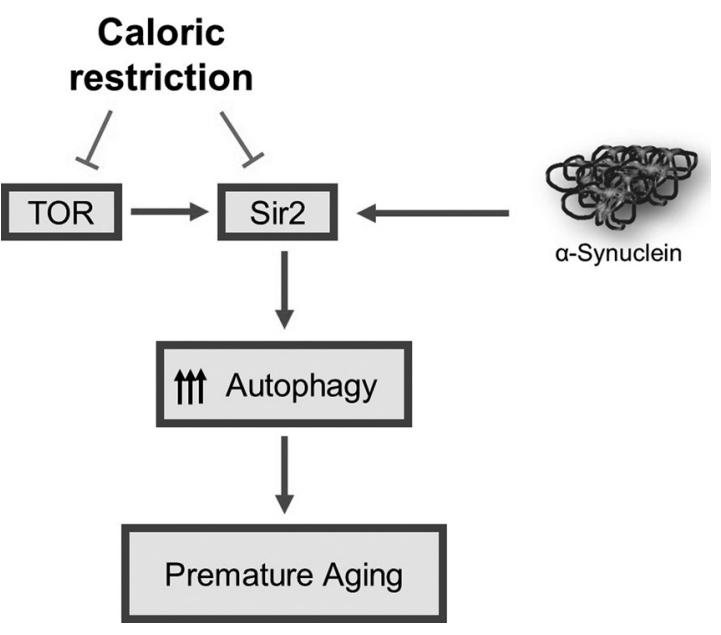

Fig. 4. The caloric restriction-mediated effects in the yeast model for synucleinopathies - work model. Caloric restriction intervention inhibits Tor1 and Sir2 activities. Inhibition of these two players, particularly Sir2, which activity is elicited by $\alpha$-synuclein expression under non-caloric restriction conditions, led to the maintenance of autophagy at homeostatic levels and consequently to aging delay.

2000; Rogina and Helfand, 2004). Nevertheless, the link between CR-induced longevity and activation of sirtuins remains tenuous. Despite the controversy around the role of Sir2 in yeast replicative lifespan and CLS and its contribution to CR-extended lifespan, Sir2 is able to modulate autophagy, and is responsible for the exacerbated autophagy elicited by syn expression (Sampaio-Marques et al., 2012). Herein, we demonstrated that in yeast cells expressing syn, CR promotes inhibition of Sir2. In addition, a crosstalk between Tor1 and Sir2 is suggested, given the data showing that TOR1 deletion leads to the down regulation of Sir2 activity. If it is well accepted that TOR inhibition is associated with the longevity effects promoted by $\mathrm{CR}$, the role of sirtuins in this context is more debatable. The idea that sirtuins mediated the longevity effects promoted by CR was challenged by a work describing the absence of effects of Sir2 overexpression on lifespan of $C$. elegans and Drosophila melanogaster models (Burnett et al., 2011). Furthermore, it is also described that Sirt1 can have distinct roles in response to CR-mimics compounds (Morselli et al., 2010; Morselli et al., 2011). Thus, in an attempt to complete this intricate puzzle, we suggest that in yeast cells expressing syn, CR intervention leads to Sir2 inhibition, which, at least, partially results in the regulation of autophagy at homeostatic levels. Particularly, data suggests that the crosstalk between TOR and Sir2 is most probably promoting the maintenance of autophagy activity at homeostatic levels (Fig. 4).

In this study, we provide evidence supporting a link between $C R$, Tor1 and Sir2 in the context of synucleinopathies using a simple model organism. The finding that CR inhibition of Tor1 and Sir2 results in homeostatic autophagy levels and rescue cells from syn toxicity opens novel avenues for intervention in synucleinopathies.

\section{Author contributions}

The experiments were conceived and designed by PL. The experiments were performed by AG and BSM. Data analysis and manuscript conception were performed by BSM and PL.

\section{Acknowledgment}

BSM is supported by the fellowship SFRH/BPD/90533/2012 funded by the Fundação para a Ciência e Tecnologia (FCT, Portugal). The research leading to these results received funding from the Fundação para a Ciência e Tecnologia (FCT), co-funded by
Programa Operacional Regional do Norte (ON.2-O Novo Norte); from the Quadro de Referência Estratégico Nacional (QREN) through the Fundo Europeu de Desenvolvimento Regional (FEDER) and from the Projeto Estratégico - LA 26-2013-2014 (PEstC/SAU/LA0026/2013).

\section{References}

Alvers, A.L., Fishwick, L.K., Wood, M.S., Hu, D., Chung, H.S., Dunn Jr., W.A., Aris, J.P., 2009. Autophagy and amino acid homeostasis are required for chronological longevity in Saccharomyces cerevisiae. Aging Cell 8, 353-369, http://dx.doi.org/ 10.1111/j.1474-9726.2009.00469.x.

Aris, J.P., Alvers, A.L., Ferraiuolo, R.A., Fishwick, L.K., Hanvivatpong, A., Hu, D., Kirlew, C., Leonard, M.T., Losin, K.J., Marraffini, M., et al., 2013. Autophagy and leucine promote chronological longevity and respiration proficiency during calorie restriction in yeast. Exp. Gerontol. 48, 1107-1119, http://dx.doi.org/10. 1016/j.exger.2013.01.006S0531-5565(13)00012-0 ([pii].).

Blagosklonny, M.V., 2010. Linking calorie restriction to longevity through sirtuins and autophagy: any role for TOR. Cell Death Dis. 1, e12, http://dx.doi.org/10. 1038/cddis.2009.17.

Boily, G., Seifert, E.L., Bevilacqua, L., He, X.H., Sabourin, G., Estey, C., Moffat, C., Crawford, S., Saliba, S., Jardine, K., et al., 2008. SirT1 regulates energy metabolism and response to caloric restriction in mice. PLoS One 3, e1759, http://dx.doi.org/10. 1371/journal.pone.0001759.

Burnett, C., Valentini, S., Cabreiro, F., Goss, M., Somogyvari, M., Piper, M.D., Hoddinott, M., Sutphin, G.L., Leko, V., McElwee, J.J., et al., 2011. Absence of effects of Sir2 overexpression on lifespan in C. elegans and Drosophila. Nature 477, 482-485, http://dx.doi.org/10.1038/nature10296.

Buttner, S., Delay, C., Franssens, V., Bammens, T., Ruli, D., Zaunschirm, S., de Oliveira, R.M., Outeiro, T.F., Madeo, F., Buee, L., et al., 2010. Synphilin-1 enhances alphasynuclein aggregation in yeast and contributes to cellular stress and cell death in a Sir2-dependent manner. PLoS One 5, e13700, http://dx.doi.org/10.1371/ journal.pone.0013700.

Cheong, H., Klionsky, D.J., 2008. Biochemical methods to monitor autophagy-related processes in yeast. Methods Enzymol. 451, 1-26, http://dx.doi.org/10.1016/ S0076-6879(08)03201-1.

Choubey, V., Safiulina, D., Vaarmann, A., Cagalinec, M., Wareski, P., Kuum, M. Zharkovsky, A., Kaasik, A., 2011. Mutant A53T alpha-synuclein induces neuronal death by increasing mitochondrial autophagy. J. Biol. Chem. 286, 10814-10824 http://dx.doi.org/10.1074/jbc.M110.132514.

Cohen, H.Y., Miller, C., Bitterman, K.J., Wall, N.R., Hekking, B., Kessler, B., Howitz, K.T., Gorospe, M., de Cabo, R., Sinclair, D.A., 2004. Calorie restriction promotes mammalian cell survival by inducing the SIRT1 deacetylase. Science 305, 390-392, http://dx.doi.org/10.1126/science.1099196.

Crews, L. Spencer, B., Desplats, P. Patrick, C., Paulino, A., Rockenstein, E., Hansen, L., Adame, A., Galasko, D., Masliah, E., 2010. Selective molecular alterations in the autophagy pathway in patients with Lewy body disease and in models of alpha-synucleinopathy. PLoS One 5, e9313, http://dx.doi.org/10.1371/journal. pone.0009313.

Cuervo, A.M., 2008. Calorie restriction and aging: the ultimate cleansing diet. J. Gerontol. A Biol. Sci. Med. Sci. 63, 547-549.

Decressac, M., Mattsson, B., Weikop, P., Lundblad, M., Jakobsson, J., Bjorklund, A., 2013. TFEB-mediated autophagy rescues midbrain dopamine neurons from alpha-synuclein toxicity. Proc. Natl. Acad. Sci. U. S. A. 110, E1817-E1826, http:// dx.doi.org/10.1073/pnas.1305623110.

Dilova, I., Easlon, E., Lin, S.J., 2007. Calorie restriction and the nutrient sensing signaling pathways. Cell. Mol. life Sci.: CMLS 64, 752-767, http://dx.doi.org/10.1007/ s00018-007-6381-y.

Donmez, G., Arun, A., Chung, C.Y., McLean, P.J., Lindquist, S., Guarente, L., 2012. SIRT1 protects against alpha-synuclein aggregation by activating molecular chaperones. J. Neurosci. 32, 124-132, http://dx.doi.org/10.1523/JNEUROSCI.3442-11. 2012.

Duan, W., Mattson, M.P., 1999. Dietary restriction and 2-deoxyglucose administration improve behavioral outcome and reduce degeneration of dopaminergic neurons in models of Parkinson's disease. J. Neurosci. Res. 57, 195-206.

Fontana, L., Partridge, L., 2015. Promoting health and longevity through diet: from model organisms to humans. Cell 161, 106-118, http://dx.doi.org/10.1016/j.cell. 2015.02.020.

Fontana, L., Partridge, L., Longo, V.D., 2010. Extending healthy life span - from yeast to humans. Science 328, 321-326, http://dx.doi.org/10.1126/science.1172539.

Franssens, V., Boelen, E., Anandhakumar, J., Vanhelmont, T., Buttner, S., Winderickx, J., 2010. Yeast unfolds the road map toward alpha-synuclein-induced cell death. Cell Death Differ. 17, 746-753, http://dx.doi.org/10.1038/cdd.2009.203.

Jadiya, P., Chatterjee, M., Sammi, S.R., Kaur, S., Palit, G., Nazir, A., 2011. Sir-2.1 modulates 'calorie-restriction-mediated' prevention of neurodegeneration in Caenorhabditis elegans: implications for Parkinson's disease. Biochem. Biophys. Res. Commun. 413, 306-310, http://dx.doi.org/10.1016/j.bbrc.2011.08.092.

Jia, K., Levine, B., 2007. Autophagy is required for dietary restriction-mediated life span extension in C. elegans. Autophagy 3, 597-599.

Kaeberlein, M., Powers 3rd., R.W., Steffen, K.K., Westman, E.A., Hu, D., Dang, N., Kerr, E.O., Kirkland, K.T., Fields, S., Kennedy, B.K., 2005. Regulation of yeast replicative life span by TOR and Sch9 in response to nutrients. Science 310, 1193-1196, http://dx.doi.org/10.1126/science.1115535. 
Lin, S.J., Defossez, P.A., Guarente, L., 2000. Requirement of NAD and SIR2 for lifespan extension by calorie restriction in Saccharomyces cerevisiae. Science 289, 2126-2128.

Liu, Y., Levine, B., 2015. Autosis and autophagic cell death: the dark side of autophagy. Cell Death Differ. 22, 367-376, http://dx.doi.org/10.1038/cdd.2014.143.

Madeo, F., Carmona-Gutierrez, D., Ring, J., Buttner, S., Eisenberg, T., Kroemer, G., 2009. Caspase-dependent and caspase-independent cell death pathways in yeast. Biochem. Biophys. Res. Commun. 382, 227-231, http://dx.doi.org/10.1016/j. bbrc.2009.02.117.

Mari, M., Griffith, J., Rieter, E., Krishnappa, L., Klionsky, D.J., Reggiori, F., 2010. An Atg9-containing compartment that functions in the early steps of autophagosome biogenesis. J. Cell Biol. 190, 1005-1022, http://dx.doi.org/10.1083/jcb. 200912089.

Maswood, N., Young, J., Tilmont, E., Zhang, Z., Gash, D.M., Gerhardt, G.A., Grondin, R., Roth, G.S., Mattison, J., Lane, M.A., et al., 2004. Caloric restriction increases neurotrophic factor levels and attenuates neurochemical and behavioral deficits in a primate model of Parkinson's disease. Proc. Natl. Acad. Sci. U. S. A. 101, 18171-18176, http://dx.doi.org/10.1073/pnas.0405831102.

Medvedik, O., Lamming, D.W., Kim, K.D., Sinclair, D.A., 2007. MSN2 and MSN4 link calorie restriction and TOR to sirtuin-mediated lifespan extension in Saccharomyces cerevisiae. PLoS Biol. 5, e261, http://dx.doi.org/10.1371/journal.pbio. 0050261.

Mendl, N., Occhipinti, A., Muller, M., Wild, P., Dikic, I., Reichert, A.S., 2011. Mitophagy in yeast is independent of mitochondrial fission and requires the stress response gene WHI2. J. Cell Sci. 124, 1339-1350, http://dx.doi.org/10.1242/jcs.076406.

Mesquita, A., Weinberger, M., Silva, A., Sampaio-Marques, B., Almeida, B., Leao, C., Costa, V., Rodrigues, F., Burhans, W.C., Ludovico, P., 2010. Caloric restriction or catalase inactivation extends yeast chronological lifespan by inducing $\mathrm{H} 2 \mathrm{O} 2$ and superoxide dismutase activity. Proc. Nat. Acad. Sci. U. S. A. 107, 15123-15128, http://dx.doi.org/10.1073/pnas.1004432107.

Mladenovic, A., Perovic, M., Tanic, N., Petanceska, S., Ruzdijic, S., Kanazir, S., 2007. Dietary restriction modulates alpha-synuclein expression in the aging rat cortex and hippocampus. Synapse 61, 790-794, http://dx.doi.org/10.1002/syn.20427.

Morselli, E., Maiuri, M.C., Markaki, M., Megalou, E., Pasparaki, A., Palikaras, K., Criollo, A., Galluzzi, L., Malik, S.A., Vitale, I., et al., 2010. Caloric restriction and resveratrol promote longevity through the Sirtuin-1-dependent induction of autophagy. Cell Death Dis. 1, e10, http://dx.doi.org/10.1038/cddis.2009.8.

Morselli, E., Marino, G., Bennetzen, M.V., Eisenberg, T., Megalou, E., Schroeder, S., Cabrera, S., Benit, P., Rustin, P., Criollo, A., et al., 2011. Spermidine and resveratrol induce autophagy by distinct pathways converging on the acetylproteome. J. Cell Biol. 192, 615-629, http://dx.doi.org/10.1083/jcb.201008167.

Noda, T., Klionsky, D.J., 2008. The quantitative Pho8Delta60 assay of nonspecific autophagy. Methods Enzymol. 451, 33-42, http://dx.doi.org/10.1016/S00766879(08)03203-5.

Outeiro, T.F., Lindquist, S., 2003. Yeast cells provide insight into alpha-synuclein biology and pathobiology. Science 302, 1772-1775, http://dx.doi.org/10.1126/ science.1090439.
Rogina, B., Helfand, S.L., 2004. Sir2 mediates longevity in the fly through a pathway related to calorie restriction. Proc. Natl. Acad. Sci. U. S. A. 101, 15998-16003, http://dx.doi.org/10.1073/pnas.0404184101.

Sampaio-Marques, B., Felgueiras, C., Silva, A., Rodrigues, F., Ludovico, P., 2011. Yeast chronological lifespan and proteotoxic stress: is autophagy good or bad? Biochem. Soc. Trans. 39, 1466-1470, http://dx.doi.org/10.1042/BST0391466.

Sampaio-Marques, B., Felgueiras, C., Silva, A., Rodrigues, M., Tenreiro, S., Franssens, V., Reichert, A.S., Outeiro, T.F., Winderickx, J., Ludovico, P., 2012. SNCA (alpha-synuclein)-induced toxicity in yeast cells is dependent on sirtuin 2(Sir2)mediated mitophagy. Autophagy 8, 1494-1509, http://dx.doi.org/10.4161/auto. 21275.

Sampaio-Marques, B., Burhans, W.C., Ludovico, P., 2014. Longevity pathways and maintenance of the proteome: the role of autophagy and mitophagy during yeast ageing. Microb. Cell 1, 118-127.

Speakman, J.R., Mitchell, S.E., 2011. Caloric restriction. Mol. Aspects Med. 32 159-221, http://dx.doi.org/10.1016/j.mam.2011.07.001.

Spillantini, M.G., Crowther, R.A., Jakes, R., Hasegawa, M., Goedert, M., 1998. alphaSynuclein in filamentous inclusions of Lewy bodies from Parkinson's disease and dementia with lewy bodies. Proc. Natl. Acad. Sci. U. S. A. 95, 6469-6473.

Stefanis, L., Larsen, K.E., Rideout, H.J., Sulzer, D., Greene, L.A., 2001. Expression of A53T mutant but not wild-type alpha-synuclein in PC12 cells induces alterations of the ubiquitin-dependent degradation system, loss of dopamine release, and autophagic cell death. J. Neurosci. 21, 9549-9560.

Tenreiro, S., Munder, M.C., Alberti, S., Outeiro, T.F., 2013. Harnessing the power of yeast to unravel the molecular basis of neurodegeneration. J. Neurochem. 127, 438-452, http://dx.doi.org/10.1111/jnc.12271.

Vaquero, A., 2009. The conserved role of sirtuins in chromatin regulation. Int. J. Dev Biol. 53, 303-322, http://dx.doi.org/10.1387/ijdb.082675av.

Vartiainen, S., Aarnio, V., Lakso, M., Wong, G., 2006. Increased lifespan in transgenic Caenorhabditis elegans overexpressing human alpha-synuclein. Exp. Gerontology 41, 871-876, http://dx.doi.org/10.1016/j.exger.2006.05.005.

Wood, J.G., Rogina, B., Lavu, S., Howitz, K., Helfand, S.L., Tatar, M., Sinclair, D. 2004. Sirtuin activators mimic caloric restriction and delay ageing in metazoans. Nature 430, 686-689, http://dx.doi.org/10.1038/nature02789.

Xilouri, M., Vogiatzi, T., Vekrellis, K., Park, D., Stefanis, L., 2009. Abberant alpha-synuclein confers toxicity to neurons in part through inhibition of chaperone-mediated autophagy. PLoS One 4, e5515, http://dx.doi.org/10.1371/ journal.pone.0005515.

Yeger-Lotem, E., Riva, L., Su, L.J., Gitler, A.D., Cashikar, A.G., King, O.D., Auluck, P.K. Geddie, M.L., Valastyan, J.S., Karger, D.R., et al., 2009. Bridging high-throughput genetic and transcriptional data reveals cellular responses to alpha-synuclein toxicity. Nat. Genet. 41, 316-323, http://dx.doi.org/10.1038/ng.337.

Zhang, T., Lei, J., Yang, H., Xu, K., Wang, R., Zhang, Z., 2011. An improved method for whole protein extraction from yeast Saccharomyces cerevisiae. Yeast 28 , 795-798, http://dx.doi.org/10.1002/yea.1905.

van Ham, T.J., Thijssen, K.L., Breitling, R., Hofstra, R.M., Plasterk, R.H., Nollen, E.A. 2008. C. elegans model identifies genetic modifiers of alpha-synuclein inclusion formation during aging. PLoS Genet. 4, e1000027, http://dx.doi.org/10.1371/ journal.pgen.1000027. 